\title{
Characterization of low pass filters in a multiresolution analysis
}

by

\author{
A. San Antolín (Madrid)
}

\begin{abstract}
We characterize the low pass filters associated with scaling functions of a multiresolution analysis in a general context, where instead of the dyadic dilation one considers the dilation given by a fixed linear invertible map $A: \mathbb{R}^{n} \rightarrow \mathbb{R}^{n}$ such that $A\left(\mathbb{Z}^{n}\right) \subset \mathbb{Z}^{n}$ and all (complex) eigenvalues of $A$ have modulus greater than 1 . This characterization involves the notion of filter multiplier of such a multiresolution analysis. Moreover, the paper contains a characterization of the measurable functions which are filter multipliers.
\end{abstract}

1. Introduction. A multiresolution analysis (MRA) is a general method introduced by Mallat [20] and Meyer [21] for constructing wavelets. Afterwards, the concept of MRA was considered on $L^{2}\left(\mathbb{R}^{n}\right), n \geq 1$, (see [19], [10], [24], [25]) in a more general context, where instead of the dyadic dilation one considers the dilation given by a fixed linear invertible map $A: \mathbb{R}^{n} \rightarrow \mathbb{R}^{n}$ such that $A\left(\mathbb{Z}^{n}\right) \subset \mathbb{Z}^{n}$ and all (complex) eigenvalues of $A$ have modulus greater than 1 . Here and further we use the same notation for the linear invertible map $A$ and its matrix with respect to the canonical basis. Given such a linear invertible map $A$ one defines an $A$-MRA as a sequence of closed subspaces $V_{j}, j \in \mathbb{Z}$, of the Hilbert space $L^{2}\left(\mathbb{R}^{n}\right)$ that satisfies the following conditions:

(i) for all $j \in \mathbb{Z}, V_{j} \subset V_{j+1}$;

(ii) for all $j \in \mathbb{Z}, f(\mathbf{x}) \in V_{j} \Leftrightarrow f(A \mathbf{x}) \in V_{j+1}$;

(iii) $\overline{\bigcup_{j \in \mathbb{Z}} V_{j}}=L^{2}\left(\mathbb{R}^{n}\right)$;

(iv) there exists a function $\phi \in V_{0}$, called a scaling function, such that $\left\{\phi(\mathbf{x}-\mathbf{k}): \mathbf{k} \in \mathbb{Z}^{n}\right\}$ is an orthonormal basis for $V_{0}$.

2000 Mathematics Subject Classification: Primary 42C40; Secondary 47B38, 42B15, 42B35, 28A33.

Key words and phrases: approximate continuity, filter multiplier, Fourier transform, low pass filter, multiresolution analysis.

The author was partially supported by \#MTM 2004-00678. 
Properties of scaling functions have been studied by several authors (see [20], [15], [8], [10], [1], [7], [13], [18], [4]).

In this paper, the Fourier transform of a function $f \in L^{1}\left(\mathbb{R}^{n}\right) \cap L^{2}\left(\mathbb{R}^{n}\right)$ is defined by

$$
\widehat{f}(\mathbf{y})=\int_{\mathbb{R}^{n}} f(\mathbf{x}) e^{-2 \pi i \mathbf{x} \cdot \mathbf{y}} d \mathbf{x} .
$$

If $\phi$ is a scaling function of an $A$-MRA, observe that $d_{A}^{-1} \phi\left(A^{-1} \mathbf{x}\right) \in$ $V_{-1} \subset V_{0}$, where $d_{A}=|\operatorname{det} A|$. By condition (iv) we express this function in terms of the orthonormal basis $\left\{\phi(\mathbf{x}-\mathbf{k}): \mathbf{k} \in \mathbb{Z}^{n}\right\}$ as

$$
d_{A}^{-1} \phi\left(A^{-1} \mathbf{x}\right)=\sum_{\mathbf{k} \in \mathbb{Z}^{n}} a_{\mathbf{k}} \phi(\mathbf{x}-\mathbf{k}),
$$

where the convergence is in $L^{2}\left(\mathbb{R}^{n}\right)$ and $\left\{a_{\mathbf{k}}\right\}_{\mathbf{k} \in \mathbb{Z}^{n}} \in l^{2}$. Taking the Fourier transform, we obtain

$$
\widehat{\phi}\left(A^{*} \mathbf{t}\right)=H(\mathbf{t}) \widehat{\phi}(\mathbf{t}) \quad \text { a.e. on } \mathbb{R}^{n}
$$

where $A^{*}$ is the adjoint map of $A$ and

$$
H(\mathbf{t})=\sum_{\mathbf{k} \in \mathbb{Z}^{n}} a_{\mathbf{k}} e^{-2 \pi i \mathbf{k} \cdot \mathbf{t}}
$$

is a $\mathbb{Z}^{n}$-periodic function which is called the low pass filter associated with the scaling function $\phi$. In this paper we are going to characterize the low pass filters associated with scaling functions of an $A$-MRA.

Before formulating our results let us introduce some notation.

Let $\mathbb{T}^{n}=\mathbb{R}^{n} / \mathbb{Z}^{n}$. Writing $F \in L^{2}\left(\mathbb{T}^{n}\right)$ we understand that $F$ is defined on the whole space $\mathbb{R}^{n}$ as a $\mathbb{Z}^{n}$-periodic function. With some abuse of notation we also consider that $\mathbb{T}^{n}$ is the unit cube $[0,1)^{n}$.

We set $B_{r}(\mathbf{y})=\left\{\mathbf{x} \in \mathbb{R}^{n}:|\mathbf{x}-\mathbf{y}|<r\right\}$, and write $B_{r}$ if $\mathbf{y}$ is the origin. For $E \subset \mathbb{R}^{n}$ and $a \in \mathbb{R}$ we define $a E=\left\{\mathbf{x} \in \mathbb{R}^{n}: \mathbf{x}=a \mathbf{t}\right.$ for some $\left.\mathbf{t} \in E\right\}$. If $\mathbf{x} \in \mathbb{R}^{n}$ then $\mathbf{x}+E=\{\mathbf{x}+\mathbf{y}: \mathbf{y} \in E\}$. The Lebesgue measure of $E \subset \mathbb{R}^{n}$ is denoted by $|E|_{n}$.

In [4] the following definitions were introduced.

Definition 1 . We say that $\mathbf{x} \in \mathbb{R}^{n}$ is a point of $A$-density for a set $E \subset \mathbb{R}^{n}$ with $|E|_{n}>0$ if for any $r>0$,

$$
\lim _{j \rightarrow \infty} \frac{\left|E \cap\left(A^{-j} B_{r}+\mathbf{x}\right)\right|_{n}}{\left|A^{-j} B_{r}\right|_{n}}=1 .
$$

Definition 2. Let $f: \mathbb{R}^{n} \rightarrow \mathbb{C}$ be a measurable function. We say that $\mathbf{x} \in \mathbb{R}^{n}$ is a point of $A$-approximate continuity of $f$ if there exists $E \subset \mathbb{R}^{n}$ with $|E|_{n}>0$ such that $\mathbf{x}$ is a point of $A$-density for $E$ and

$$
\lim _{\substack{\mathbf{y} \rightarrow \mathbf{x} \\ \mathbf{y} \in E}} f(\mathbf{y})=f(\mathbf{x}) \text {. }
$$


Definition 3. A measurable function $f: \mathbb{R}^{n} \rightarrow \mathbb{C}$ is said to be $A$-locally nonzero at $\mathbf{x} \in \mathbb{R}^{n}$ if for any $\varepsilon, r>0$ there exists $j \in \mathbb{N}$ such that

$$
\left|\left\{\mathbf{y} \in A^{-j} B_{r}+\mathbf{x}: f(\mathbf{y})=0\right\}\right|_{n}<\varepsilon\left|A^{-j} B_{r}\right|_{n} .
$$

Observe that if $A=a I$, where $a>1$ and $I$ is the identity map, the definition of a point of $A$-approximate continuity coincides with the well known definition of approximate continuity (cf. [22], [3]).

For a given $\phi \in L^{2}\left(\mathbb{R}^{n}\right)$, set

$$
\Phi_{\phi}(\mathbf{t})=\sum_{\mathbf{k} \in \mathbb{Z}^{n}}|\widehat{\phi}(\mathbf{t}+\mathbf{k})|^{2} .
$$

If $A: \mathbb{R}^{n} \rightarrow \mathbb{R}^{n}$ is a linear invertible map such that $A\left(\mathbb{Z}^{n}\right) \subset \mathbb{Z}^{n}$ and all (complex) eigenvalues of $A$ have modulus greater than 1 , recall that a coset of $A\left(\mathbb{Z}^{n}\right)$ is a set of the form

$$
\mathbf{q}+A\left(\mathbb{Z}^{n}\right)=\left\{\mathbf{q}+A \mathbf{k}: \mathbf{k} \in \mathbb{Z}^{n}\right\}
$$

where $\mathbf{q}$ is any element of $\mathbb{Z}^{n}$ which is sometimes referred to as a representative of the coset. Any pair of cosets are either identical or disjoint so that the collection of all cosets, denoted by $\mathbb{Z}^{n} / A\left(\mathbb{Z}^{n}\right)$, consists of disjoint cosets whose union is $\mathbb{Z}^{n}$. We have $\operatorname{card}\left(\mathbb{Z}^{n} / A\left(\mathbb{Z}^{n}\right)\right)=\operatorname{card}\left(\mathbb{Z}^{n} / A^{*}\left(\mathbb{Z}^{n}\right)\right)=d_{A} \geq 2$ (see [10] and [25, p. 109]). A subset $\Delta_{A}$ of $\mathbb{Z}^{n}$ is said to be a full collection of representatives of $\mathbb{Z}^{n} / A\left(\mathbb{Z}^{n}\right)$ if it contains exactly $d_{A}$ elements and

$$
\bigcup_{\mathbf{q} \in \Delta_{A}}\left(\mathbf{q}+A\left(\mathbb{Z}^{n}\right)\right)=\mathbb{Z}^{n}
$$

Let us fix $\Delta_{A}=\left\{\mathbf{q}_{i}\right\}_{i=0}^{d_{A}-1}$ and $\Delta_{A^{*}}=\left\{\mathbf{p}_{i}\right\}_{i=0}^{d_{A}-1}$, where $\mathbf{q}_{0}=\mathbf{p}_{0}=\mathbf{0}$.

Given $H \in L^{\infty}\left(\mathbb{T}^{n}\right)$, the continuous linear operator $P: L^{1}\left(\mathbb{T}^{n}\right) \rightarrow L^{1}\left(\mathbb{T}^{n}\right)$ with

$$
P f(\mathbf{t})=\sum_{i=0}^{d_{A}-1}\left|H\left(\left(A^{*}\right)^{-1}\left(\mathbf{t}+\mathbf{p}_{i}\right)\right)\right|^{2} f\left(\left(A^{*}\right)^{-1}\left(\mathbf{t}+\mathbf{p}_{i}\right)\right)
$$

is well defined. This operator was first introduced by M. Bownik [2] as a generalization of the analogous operator introduced by W. Lawton [17] for dyadic dilations.

For the study of functions $H \in L^{\infty}\left(\mathbb{T}^{n}\right)$ which give rise to a scaling function of an $A$-MRA suppose that the infinite product

$$
\prod_{j=1}^{\infty}\left|H\left(\left(A^{*}\right)^{-j} \mathbf{t}\right)\right|
$$


converges almost everywhere on $\mathbb{R}^{n}$. We are going to look for a scaling function $\phi$ of an $A$-MRA which satisfies the condition

$$
|\widehat{\phi}(\mathbf{t})|=\prod_{j=1}^{\infty}\left|H\left(\left(A^{*}\right)^{-j} \mathbf{t}\right)\right| .
$$

Hence, according to the properties of the scaling functions of an $A$-MRA (see Theorem A below), we should also suppose that $|\widehat{\phi}|$ is $A^{*}$-locally nonzero at the origin. In order not to repeat those conditions let us introduce the class $\mathbf{H}_{A}$ of all functions $H \in L^{\infty}\left(\mathbb{T}^{n}\right)$ such that the infinite product (2) converges almost everywhere on $\mathbb{R}^{n}$ and is $A^{*}$-locally nonzero at the origin.

Moreover, let us introduce the class $\Pi_{A}$ of all measurable functions $f$ on $\mathbb{R}^{n}$ such that $0 \leq f(\mathbf{t}) \leq 1$ a.e. on $\mathbb{R}^{n}$ and the origin is a point of $A^{*}$-approximate continuity of $f$ if we set $f(\mathbf{0})=1$.

We prove the following.

Theorem 1. Let $H \in \mathbf{H}_{A}$. Then the following conditions are equivalent:

(A) The function $|H|$ is a low pass filter associated with a scaling function $\theta$ of an $A-M R A$ where $\widehat{\theta}(\mathbf{t}):=\prod_{j=1}^{\infty}\left|H\left(\left(A^{*}\right)^{-j} \mathbf{t}\right)\right|$.

(B) The only function $f \in L^{1}\left(\mathbb{T}^{n}\right) \cap \Pi_{A}$ invariant under the operator $P$ is the function $f \equiv 1$.

To give a complete characterization of all low pass filters associated with scaling functions, we need the notion of a filter multiplier which was introduced in [26] for the one-dimensional case.

Definition 4. We say that a measurable function $m$ is a filter multiplier if whenever $H$ is a low pass filter associated with a scaling function of an $A$-MRA, then $m H$ is a low pass filter associated with a scaling function of some $A$-MRA.

In the above definition we do not use the term $A$-filter multiplier because as will be seen in Theorem 2, the class of filter multipliers is the same for all linear invertible maps $A: \mathbb{R}^{n} \rightarrow \mathbb{R}^{n}$ such that all eigenvalues of $A$ have modulus greater than 1 and $A\left(\mathbb{Z}^{n}\right) \subset \mathbb{Z}^{n}$. The following result generalizes a similar assertion for the one-dimensional case (see [26]).

TheOREM 2. A measurable function $m$ is a filter multiplier if and only if $m$ is a $\mathbb{Z}^{n}$-periodic function and $|m(\mathbf{t})|=1$ a.e. on $\mathbb{R}^{n}$.

REMARK 1. According to Theorem 2, a measurable function $H$ is a low pass filter of an $A$-MRA if and only if $|H|$ is a low pass filter of some $A$-MRA. Indeed, in the proof of Theorem 2 it will be shown that if $H \in L^{\infty}\left(\mathbb{T}^{n}\right)$ is such that $|H|$ is a low pass filter associated with a scaling function $\theta$ of an $A$-MRA, then $H$ is a low pass filter associated with a scaling function $\varphi$ of 
some $A$-MRA defined by $\widehat{\varphi}=\mu \widehat{\theta}$ where $\mu$ is any measurable function defined on $\mathbb{R}^{n}$ which satisfies

$$
|\mu(\mathbf{t})|=1 \quad \text { a.e. on } \mathbb{R}^{n} \quad \text { and } \quad m_{H}(\mathbf{t})=\mu\left(A^{*} \mathbf{t}\right) \overline{\mu(\mathbf{t})}
$$

where

$$
m_{H}(\mathbf{t})= \begin{cases}H(\mathbf{t}) /|H(\mathbf{t})| & \text { if }|H(\mathbf{t})| \neq 0 \\ 1 & \text { if }|H(\mathbf{t})|=0\end{cases}
$$

Historically, several sufficient conditions are known such that for a given function $H$, the infinite product $\widehat{\phi}(t)=\prod_{j=1}^{\infty} H\left(2^{-j} t\right)$ exists a.e. on $\mathbb{R}$ and $\phi$ is a scaling function of an MRA on $L^{2}(\mathbb{R})$.

A. Cohen [5] gave the first necessary and sufficient conditions for a trigonometric polynomial $H$ to be a low pass filter of an MRA on $L^{2}(\mathbb{R})$. Cohen's conditions may be viewed as geometric restrictions on $H$. Afterwards, Cohen's approach was developed by E. Hernández and G. Weiss [13], M. Papadakis, H. Sikić and G. Weiss [23] and R. F. Gundy [11]. About the same time as Cohen's condition appeared, W. Lawton [16] gave another sufficient condition of a different nature when $H$ is a trigonometric polynomial. The necessity of Lawton's condition was settled in 1990 by Cohen [6] and Lawton [17] independently (see [8, pp. 182-193]).

For our general case when the MRA is defined on $L^{2}\left(\mathbb{R}^{n}\right), n \geq 1$, and for dilations given by a map $A$ as described above, a generalization of Cohen's conditions for low pass filters associated with characteristic scaling functions was proved by K. Gröchenig and W. R. Madych [10] and W. R. Madych [19]. Afterwards, a generalization of Cohen's and Lawton's conditions was obtained by M. Bownik [2].

The problem of when a given function $H \in L^{\infty}\left(\mathbb{T}^{n}\right)$ is a low pass filter for an MRA was posed in the book by E. Hernández and G. Weiss [13].

Characterizations of low pass filters for an MRA on $L^{2}(\mathbb{R})$ are already known: see the papers by M. Papadakis, H. Sikić and G. Weiss [23] and by V. Dobrić, R. F. Gundy and P. Hitczenko [9]. Afterwards, R. F. Gundy [12] addressed the same question when condition (iv) in the definition of MRA is relaxed by assuming that $\{\phi(x-k): k \in \mathbb{Z}\}$ is a Riesz basis for $V_{0}$.

Note that the conditions presented here follow the strategy of Lawton and are new even in the classical case, i.e., for an MRA on $L^{2}(\mathbb{R})$ and the dyadic dilations.

The key tool for the proof of Theorem 1 is the characterization of the scaling functions given in [4] which we formulate in Section 2. In that section we also give some additional well known properties of low pass filters. In Section 3 results relating to $A$-approximate continuity are presented. Section 4 is dedicated to the study of properties of the low pass filters. Finally, the proofs of Theorems 1 and 2 are given in Sections 5 and 6 respectively. 
2. Auxiliary results. The following characterization of scaling functions in a multiresolution analysis was given in [4].

Theorem A. Let $\phi \in L^{2}\left(\mathbb{R}^{n}\right)$. Then the following conditions are equivalent:

(A) The function $\phi$ is a scaling function of an A-MRA.

(B) $(\alpha)$ The function $\widehat{\phi}$ is $A^{*}$-locally nonzero at the origin;

$(\beta) \Phi_{\phi}(\mathbf{t})=1$ a.e. on $\mathbb{T}^{n}$

$(\gamma)$ There exists a $\mathbb{Z}^{n}$-periodic function $H \in L^{\infty}\left(\mathbb{T}^{n}\right)$ with $|H(\mathbf{t})| \leq 1$ a.e. on $\mathbb{R}^{n}$ such that

$$
\widehat{\phi}\left(A^{*} \mathbf{t}\right)=H(\mathbf{t}) \widehat{\phi}(\mathbf{t}) \quad \text { a.e. on } \mathbb{R}^{n} .
$$

(C) Conditions $\left(\alpha^{*}\right),(\beta)$ and $(\gamma)$ hold, where

$\left(\alpha^{*}\right)$ If we set $|\widehat{\phi}(\mathbf{0})|=1$, the origin is a point of $A^{*}$-approximate continuity of $|\widehat{\phi}|$.

For low pass filters associated with a scaling function of an $A$-MRA the following proposition is true (cf. [20], [21], [8], [13], [2]).

Proposition B. Let $H$ be a low pass filter associated with a scaling function of an $A-M R A$. Then

$$
\sum_{i=0}^{d_{A}-1}\left|H\left(\mathbf{t}+\left(A^{*}\right)^{-1} \mathbf{p}_{i}\right)\right|^{2}=1 \quad \text { a.e. on } \mathbb{R}^{n} .
$$

The following proposition was proved in [2] (cf. [8]).

Proposition C. Let $H \in L^{\infty}\left(\mathbb{T}^{n}\right)$ be a function such that (3) holds. If the infinite product $\prod_{j=1}^{\infty}\left|H\left(\left(A^{*}\right)^{-j} \mathbf{t}\right)\right|$ converges almost everywhere, then $\widehat{\theta}(\mathbf{t}):=\prod_{j=1}^{\infty}\left|H\left(\left(A^{*}\right)^{-j} \mathbf{t}\right)\right|$ belongs to $L^{2}\left(\mathbb{R}^{n}\right)$ and $\|\widehat{\theta}\|_{L^{2}\left(\mathbb{R}^{n}\right)} \leq 1$.

In the proof of Theorem 1, we will need the following technical result from [4]. Note that the equality (ii) in the following lemma does not appear in the original result but it is a direct consequence of the proof of (i).

Lemma D. Let $g \in L^{2}\left(\mathbb{T}^{n}\right)$, let $A: \mathbb{R}^{n} \rightarrow \mathbb{R}^{n}$ be a fixed linear invertible map such that $A\left(\mathbb{Z}^{n}\right) \subset \mathbb{Z}^{n}$ and let $\hat{A}: \mathbb{T}^{n} \rightarrow \mathbb{T}^{n}$ be the induced endomorphism. Then

(i) $\int_{\mathbb{T}^{n}} g(\hat{A} \mathbf{t}) d \mathbf{t}=\int_{\mathbb{T}^{n}} g(\mathbf{t}) d \mathbf{t}$.

(ii) $\int_{[0,1]^{n}} g(\mathbf{t}) d \mathbf{t}=d_{A}^{-1} \int_{[0,1]^{n}} \sum_{i=0}^{d_{A}-1} g\left(A^{-1} \mathbf{t}+A^{-1} \mathbf{p}_{i}\right) d \mathbf{t}$.

The following lemma is proved in [2] (cf. [8], [13]).

Lemma E. Let $H \in L^{\infty}\left(\mathbb{T}^{n}\right)$ be such that (3) holds. For every $N \in \mathbb{N}$ let 


$$
\Gamma_{N}(\mathbf{t})=\prod_{j=1}^{N}\left|H\left(\left(A^{*}\right)^{-j} \mathbf{t}\right)\right| \chi_{[-1 / 2,1 / 2]^{n}}\left(\left(A^{*}\right)^{-N} \mathbf{t}\right), \quad \mathbf{t} \in \mathbb{R}^{n}
$$

Then

$$
\sum_{\mathbf{k} \in \mathbb{Z}}\left|\Gamma_{N}(\mathbf{t}+\mathbf{k})\right|^{2}=1 \quad \text { a.e. on } \mathbb{R}^{n} .
$$

To give a characterization of the filter multipliers, we will need the following lemma proved by Gröchenig and Madych [10] (see also [19]).

Lemma F. Let $A: \mathbb{R}^{n} \rightarrow \mathbb{R}^{n}$ be a linear invertible map such that $A\left(\mathbb{Z}^{n}\right) \subset \mathbb{Z}^{n}$ and all (complex) eigenvalues of $A$ have modulus greater than 1 . Then any integrable solution of

$$
\phi(\mathbf{x})=\sum_{\mathbf{q} \in \Delta_{A}} \phi(A \mathbf{x}-\mathbf{q})
$$

is unique up to multiplication by a constant and is compactly supported with the compact support

$$
Q=\left\{\mathbf{x} \in \mathbb{R}^{n}: \mathbf{x}=\sum_{j=1}^{\infty} A^{-j} \mathbf{b}_{j}, \mathbf{b}_{j} \in \Delta_{A}\right\} .
$$

If $\phi_{h}$ is a compactly supported function which satisfies (4), then by the well known Paley-Wiener-Schwartz Theorem (see [14, p. 181]) we know that $\left|\left\{\mathbf{t} \in \mathbb{R}^{n}: \widehat{\phi}_{h}(\mathbf{t})=0\right\}\right|_{n}=0$. Thus if we take $\widehat{\varphi}=\widehat{\phi}_{h}\left(\Phi_{\phi_{h}}\right)^{-1 / 2}$, where $\Phi_{\phi_{h}}$ is defined by (1), then $\varphi$ will be a scaling function of an $A$-MRA (see [1, Section 2]) and the following claim is true:

Claim 1. Let $A: \mathbb{R}^{n} \rightarrow \mathbb{R}^{n}$ be a linear invertible map such that $A\left(\mathbb{Z}^{n}\right) \subset$ $\mathbb{Z}^{n}$ and all (complex) eigenvalues of $A$ have modulus greater than 1 . Then there exists a scaling function, $\varphi$, of an $A-M R A$ such that the support of the low pass filter $H$ associated with $\varphi$ coincides a.e. with $\mathbb{R}^{n}$, i.e.

$$
\left|\left\{\mathbf{t} \in \mathbb{R}^{n}: H(\mathbf{t})=0\right\}\right|_{n}=0 .
$$

3. Some auxiliary results on $A$-approximate continuity. First of all, we are going to study some properties related to the concept of a point of $A$-approximate continuity which will be used in the proof of Theorem 1 .

Proposition 1. Let $A: \mathbb{R}^{n} \rightarrow \mathbb{R}^{n}$ be a linear invertible map such that all (complex) eigenvalues of $A$ have modulus greater than 1 . Let $f: \mathbb{R}^{n} \rightarrow \mathbb{C}$ be a measurable function such that for a point $\mathbf{y} \in \mathbb{R}^{n}$ we have

$$
\lim _{j \rightarrow \infty} f\left(A^{-j} \mathbf{x}+\mathbf{y}\right)=f(\mathbf{y}) \quad \text { a.e. on } \mathbb{R}^{n} .
$$

Then $\mathbf{y}$ is a point of A-approximate continuity of $f$. 
Proof. We can assume that $\mathbf{y}=\mathbf{0}$ and $f(\mathbf{0})=0$. Fix $\varepsilon>0$. For any $j, N \in \mathbb{N}$ we define

$$
F_{j}^{\varepsilon}=\left\{\mathbf{x} \in B_{1}:\left|f\left(A^{-j} \mathbf{x}\right)\right|<\varepsilon\right\}, \quad E_{N}^{\varepsilon}=\bigcap_{j \geq N} F_{j}^{\varepsilon} .
$$

By Egorov's Theorem it follows that

$$
\lim _{N \rightarrow \infty}\left|E_{N}^{\varepsilon}\right|_{n}=\left|B_{1}\right|_{n}
$$

Furthermore, obviously $E_{N}^{\varepsilon} \subset F_{N}^{\varepsilon}$ and $F_{N}^{\varepsilon} \subset B_{1}$. Then

$$
\begin{aligned}
1 & =\liminf _{N \rightarrow \infty} \frac{\left|E_{N}^{\varepsilon}\right|_{n}}{\left|B_{1}\right|_{n}} \leq \liminf _{N \rightarrow \infty} \frac{\left|F_{N}^{\varepsilon}\right|_{n}}{\left|B_{1}\right|_{n}} \\
& =\liminf _{N \rightarrow \infty} \frac{\left|\left\{\mathbf{x} \in A^{-N} B_{1}:|f(\mathbf{x})|<\varepsilon\right\}\right|_{n}}{\left|A^{-N} B_{1}\right|_{n}} \leq 1 .
\end{aligned}
$$

Hence

$$
\lim _{N \rightarrow \infty} \frac{\left|\left\{\mathbf{x} \in A^{-N} B_{1}:|f(\mathbf{x})|<\varepsilon\right\}\right|_{n}}{\left|A^{-N} B_{1}\right|_{n}}=1,
$$

which means that the origin is a point of $A$-approximate continuity of $f$ when $f(\mathbf{0})=0$.

The following counterexample shows that, in general, the converse of Proposition 1 is not true.

Proposition 2. There exists a measurable set $E \subset \mathbb{R}$ with $|E|>0$ such that the origin belongs to $E$ and is a point of approximate continuity of the function $\chi_{E}$ but $\lim _{j \rightarrow \infty} \chi_{E}\left(2^{-j} x\right)$ does not exist for any $x \in \mathbb{R} \backslash\{0\}$.

Proof. For any $j \in\{0,1,2, \ldots\}$ and any $k \in\left\{0, \ldots, 2^{j}-1\right\}$, let

We put

$$
\Lambda_{k}^{(j)}=\left(\frac{2^{j}+k}{2^{j}}, \frac{2^{j}+k+1}{2^{j}}\right] \text {. }
$$

$$
\Lambda_{m}=\Lambda_{k}^{(j)} \quad \text { for } m=2^{j}+k, \quad \text { and } \quad E_{1}=[0, \infty) \backslash \bigcup_{m=1}^{\infty} 2^{-m} \Lambda_{m} .
$$

Then

$$
E=E_{1} \cup\left(-E_{1}\right) .
$$

We claim that $\lim _{j \rightarrow \infty} \chi_{E}\left(2^{-j} x\right)$ exists for no $x \in \mathbb{R} \backslash\{0\}$. If $x \in(1,2]$, then there exists an increasing sequence $\left\{m_{\nu}\right\}_{\nu=1}^{\infty}$ of natural numbers such that $x \in \Lambda_{m_{\nu}}$ for all $\nu \in \mathbb{N}$. Suppose that $x \notin \Lambda_{m}$ if $m \neq m_{\nu}(\nu \in \mathbb{N})$. According to the definition of $E$,

$$
\begin{array}{rlrl}
\chi_{E}\left(2^{-m_{\nu}} x\right) & =0 & & \text { for all } \nu \in \mathbb{N}, \\
\chi_{E}\left(2^{-m} x\right)=1 & & \text { if } m \neq m_{\nu} .
\end{array}
$$

Hence $\lim _{j \rightarrow \infty} \chi_{E}\left(2^{-j} x\right)$ does not exist. 
Next, we observe that for any $x>0$, one can find $l \in \mathbb{Z}$ such that $2^{l} x \in(1,2]$. Thus the above argument can be employed for the sequence

$$
l+m_{\nu}: \nu=i_{l}, i_{l}+1, \ldots
$$

where $i_{l}$ is the smallest natural number such that $l+m_{\nu}>0$ if $\nu=i_{l}$. The case $x<0$ follows from the case $x>0$ by observing that $E$ is a symmetric set with respect to the origin.

On the other hand, to prove that the origin is a point of approximate continuity of $\chi_{E}$, it is sufficient to prove that it is a point of density for $E$. Let $l \in \mathbb{N}$. Then

$$
\begin{aligned}
\left|2^{l} E^{c} \cap(-1,1)\right| & =2\left|2^{l} E_{1}^{c} \cap(0,1)\right|=2\left|2^{l}\left(\bigcup_{m=1}^{\infty} 2^{-m} \Lambda_{m}\right) \cap(0,1)\right| \\
& =2\left|\left(\bigcup_{m=1}^{\infty} 2^{l-m} \Lambda_{m}\right) \cap(0,1)\right|=2\left|\bigcup_{m=l+1}^{\infty} 2^{l-m} \Lambda_{m}\right|,
\end{aligned}
$$

where the last equality is true because $\Lambda_{m} \subset[1,2]$ for $m \in \mathbb{N}$.

If we write $l+1=2^{j_{0}}+k_{0}$ where $j_{0} \in \mathbb{N}$ and $k_{0} \in\left\{0, \ldots, 2^{j_{0}}-1\right\}$, then

$$
\begin{aligned}
\left|2^{l} E_{1}^{c} \cap(0,1)\right| & =\left|\left(\bigcup_{k=k_{0}}^{2^{j_{0}}-1} 2^{k_{0}-1-k} \Lambda_{k}^{\left(j_{0}\right)}\right) \cup\left(\bigcup_{j=j_{0}+1}^{\infty} \bigcup_{k=0}^{2^{j}-1} 2^{2^{j_{0}}+k_{0}-1-2^{j}-k} \Lambda_{k}^{(j)}\right)\right| \\
& \leq \sum_{k=k_{0}}^{2^{j_{0}-1}} 2^{k_{0}-1-k}\left|\Lambda_{0}^{\left(j_{0}\right)}\right|+\sum_{j=j_{0}+1}^{\infty} 2^{2^{j_{0}}+k_{0}-1-2^{j}} \sum_{k=0}^{2^{j}-1} 2^{-k}\left|\Lambda_{0}^{(j)}\right| \\
& \leq\left|\Lambda_{0}^{\left(j_{0}\right)}\right|+\sum_{j=j_{0}+1}^{\infty} 2^{2^{j_{0}+1}-1-2^{j}}\left|\Lambda_{0}^{\left(j_{0}\right)}\right| \leq 2\left|\Lambda_{0}^{\left(j_{0}\right)}\right|=2^{-j_{0}+1} .
\end{aligned}
$$

Hence,

$$
\lim _{l \rightarrow \infty}\left|2^{l} E^{c} \cap(-1,1)\right|=0,
$$

i.e., the origin is a point of density for $E$.

In spite of the above negative result, the following proposition holds.

Proposition 3. Let $A: \mathbb{R}^{n} \rightarrow \mathbb{R}^{n}$ be a linear invertible map such that all (complex) eigenvalues of $A$ have modulus greater than 1 . Let $f: \mathbb{R}^{n} \rightarrow \mathbb{C}$ be a measurable function and $\mathbf{y} \in \mathbb{R}^{n}$ a point of $A$-approximate continuity of $f$. Then there exists an increasing sequence $\left\{j_{k}\right\}_{k=1}^{\infty} \subset \mathbb{N}$ such that

$$
\lim _{k \rightarrow \infty} f\left(A^{-j_{k}} \mathbf{x}+\mathbf{y}\right)=f(\mathbf{y}) \quad \text { a.e. on } \mathbb{R}^{n} .
$$

Proof. We can assume that $\mathbf{y}=\mathbf{0}$ and $f(\mathbf{0})=0$. It is easy to observe that the sequence of functions $\left\{f\left(A^{-j} \mathbf{x}\right)\right\}_{j=1}^{\infty}$ tends to zero in measure on any ball $B_{r}$. Hence applying Egorov's Theorem for any $r \in \mathbb{N}$, we can find 
subsequences $\left\{j_{k}^{(r)}\right\}_{k \in \mathbb{N}} \subset\left\{j_{k}^{(r-1)}\right\}_{k \in \mathbb{N}}$ of natural numbers such that

$$
\lim _{k \rightarrow \infty} f\left(A^{-j_{k}^{(r)}} \mathbf{x}\right)=0 \quad \text { a.e. on } B_{r} \text {. }
$$

Using Cantor's diagonal method of selection we obtain

$$
\lim _{k \rightarrow \infty} f\left(A^{-j_{k}^{(k)}} \mathbf{x}\right)=0 \quad \text { a.e. on } \mathbb{R}^{n} \text {. }
$$

Proposition 4. Let $A: \mathbb{R}^{n} \rightarrow \mathbb{R}^{n}$ be a linear invertible map such that all (complex) eigenvalues of $A$ have modulus greater than 1 . Let $f: \mathbb{R}^{n} \rightarrow \mathbb{C}$ be a measurable function that is A-locally nonzero at the origin. Then there exists a strictly increasing sequence $\left\{j_{k}\right\}_{k=1}^{\infty} \subset \mathbb{N}$ such that for a.e. $\mathbf{x}$ in $\mathbb{R}^{n}$ there exists $k_{0} \in \mathbb{N}$ such that $f\left(A^{-j_{k}} \mathbf{x}\right) \neq 0$ for $k \geq k_{0}$.

Proof. As $f$ is $A$-locally nonzero at the origin, for $k=1,2, \ldots$ and $\varepsilon_{k}=2^{-k}\left|B_{k}\right|_{n}^{-1}$ there exist $j_{k} \in \mathbb{N}$ with $j_{k}>j_{k-1}$ such that

$$
\left|\left\{\mathbf{x} \in A^{-j_{k}} B_{k}: f(\mathbf{x})=0\right\}\right|_{n}<2^{-k}\left|B_{k}\right|_{n}^{-1}\left|A^{-j_{k}} B_{k}\right|_{n}
$$

or equivalently, after a corresponding change of variable,

$$
\left|\left\{\mathbf{x} \in B_{k}: f\left(A^{-j_{k}} \mathbf{x}\right)=0\right\}\right|_{n}<2^{-k} .
$$

Observe that indeed $j_{k+1}>j_{k}$, because if

$$
\inf _{0 \leq j \leq j_{k}} \frac{\left|\left\{\mathbf{x} \in A^{-j} B_{k}: f(\mathbf{x})=0\right\}\right|_{n}}{\left|A^{-j} B_{k}\right|_{n}}=0,
$$

then the support of $f$ contains (almost everywhere) an open neighbourhood of the origin, so we can choose $j_{k+1}>j_{k}$. On the other hand, if

$$
\inf _{0 \leq j \leq j_{k}} \frac{\left|\left\{\mathbf{x} \in A^{-j} B_{k}: f(\mathbf{x})=0\right\}\right|_{n}}{\left|A^{-j} B_{k}\right|_{n}}=C>0,
$$

we can take an arbitrary real number $\varepsilon, 0<\varepsilon<\inf \left\{C, 2^{-k-1}\left|B_{k+1}\right|_{n}^{-1}\right\}$, and then there exist $j_{k+1}>j_{k}$ satisfying (5).

We now establish that for almost every $\mathbf{x} \in \mathbb{R}^{n}$, there exist $k_{0} \in \mathbb{N}$ such that if $k \geq k_{0}$,

$$
f\left(A^{-j_{k}} \mathbf{x}\right) \neq 0
$$

Given $N \in \mathbb{N}$, let

$$
F_{N}=\bigcup_{k=N}^{\infty}\left\{\mathbf{x} \in B_{k}: f\left(A^{-j_{k}} \mathbf{x}\right)=0\right\}, \quad E=\bigcap_{N \geq 1} F_{N} .
$$

Since $F_{1} \supset F_{2} \supset \cdots$, it follows that $\lim _{N \rightarrow \infty}\left|F_{N}\right|_{n}=|E|_{n}$. On the other hand, from (6) it is clear that

$$
\left|F_{N}\right|_{n} \leq \sum_{k=N}^{\infty} 2^{-k}=2^{-N+1}
$$

so $\lim _{N \rightarrow \infty}\left|F_{N}\right|_{n}=0$, and hence $|E|_{n}=0$. 
It remains to verify that (7) holds for all points in $\mathbb{R}^{n} \backslash E$. Let $\mathbf{y} \in \mathbb{R}^{n} \backslash E$. Then $\mathbf{y} \notin F_{N_{0}}$ for some $N_{0} \in \mathbb{N}$. In other words,

$$
\mathbf{y} \notin\left\{\mathbf{x} \in B_{k}: f\left(A^{-j_{k}} \mathbf{x}\right)=0\right\}
$$

for all $k \geq N_{0}$, and consequently $f\left(A^{-j_{k}} \mathbf{y}\right) \neq 0$ if $k \geq N_{0}$.

4. Properties of low pass filters. The following proposition holds.

Proposition 5. Let $H$ be a low pass filter associated with a scaling function of an $A-M R A$. Then the origin is a point of $A^{*}$-approximate continuity of $|H|$ if we set $|H(\mathbf{0})|=1$, and any point $\left(A^{*}\right)^{-1} \mathbf{p}_{i}, i=1, \ldots, d_{A}-1$, is a point of $A^{*}$-approximate continuity of $|H|$ if we set $\left|H\left(\left(A^{*}\right)^{-1} \mathbf{p}_{i}\right)\right|=0$.

For the proof one only needs to use a refinement equation $\widehat{\phi}\left(A^{*} \mathbf{t}\right)=$ $H(\mathbf{t}) \widehat{\phi}(\mathbf{t})$ a.e. and the $A^{*}$-approximate continuity of $|\widehat{\phi}|$ at the origin if we set $|\widehat{\phi}(\mathbf{0})|=1$ together with Proposition B.

We also have the following proposition.

Proposition 6. Let $H$ be a low pass filter associated with a scaling function $\phi$ of an $A-M R A$. Then

$$
|\widehat{\phi}(\mathbf{t})|=\prod_{j=1}^{\infty}\left|H\left(\left(A^{*}\right)^{-j} \mathbf{t}\right)\right| \quad \text { a.e. in } \mathbb{R}^{n} .
$$

Proof. Given $N \in \mathbb{N}$, from the definition of low pass filter we have

$$
\widehat{\phi}(\mathbf{t})=\left[\prod_{j=1}^{N} H\left(\left(A^{*}\right)^{-j} \mathbf{t}\right)\right] \widehat{\phi}\left(\left(A^{*}\right)^{-N} \mathbf{t}\right) \quad \text { a.e. in } \mathbb{R}^{n} .
$$

On the other hand, according to condition $\left(\alpha^{*}\right)$ of Theorem A, the origin is a point of $A^{*}$-approximate continuity of $|\widehat{\phi}|$ if we set $|\widehat{\phi}(\mathbf{0})|=1$. Hence, by Proposition 3 there exists an increasing sequence $\left\{j_{N}\right\}_{N=1}^{\infty} \subset \mathbb{N}$ such that

$$
\lim _{N \rightarrow \infty}\left|\widehat{\phi}\left(\left(A^{*}\right)^{-j_{N}} \mathbf{t}\right)\right|=1 \quad \text { a.e. on } \mathbb{R}^{n} .
$$

Moreover, as $\left|\widehat{\phi}\left(A^{*} \mathbf{t}\right)\right| \leq|\widehat{\phi}(\mathbf{t})|$ a.e. in $\mathbb{R}^{n}$, we obtain

$$
\lim _{N \rightarrow \infty}\left|\widehat{\phi}\left(\left(A^{*}\right)^{-N} \mathbf{t}\right)\right|=1 \quad \text { a.e. in } \mathbb{R}^{n} \text {. }
$$

Hence,

$$
\lim _{N \rightarrow \infty} \prod_{j=1}^{N}\left|H\left(\left(A^{*}\right)^{-j} \mathbf{t}\right)\right|=\lim _{N \rightarrow \infty} \frac{|\widehat{\phi}(\mathbf{t})|}{\left|\widehat{\phi}\left(\left(A^{*}\right)^{-N} \mathbf{t}\right)\right|}=|\widehat{\phi}(\mathbf{t})| \quad \text { a.e. in } \mathbb{R}^{n}
$$

A version of the following proposition for $n=1$ and for the dyadic dilation appears in [23]. 
Proposition 7. Let $H \in L^{\infty}\left(\mathbb{T}^{n}\right)$ be such that (3) holds and let $\widehat{\theta}(\mathbf{t})=$ $\prod_{j=1}^{\infty}\left|H\left(\left(A^{*}\right)^{-j} \mathbf{t}\right)\right|$ a.e. on $\mathbb{R}^{n}$. Then

(i) for each $N \in \mathbb{Z}, \widehat{\theta}\left(\left(A^{*}\right)^{-N} \mathbf{t}\right) \leq \widehat{\theta}\left(\left(A^{*}\right)^{-N-1} \mathbf{t}\right)$ a.e. on $\mathbb{R}^{n}$;

(ii) the limits in the following inequalities exist a.e. on $\mathbb{R}^{n}$ and

$$
0 \leq \lim _{N \rightarrow \infty} \widehat{\theta}\left(\left(A^{*}\right)^{N} \mathbf{t}\right) \leq \widehat{\theta}(\mathbf{t}) \leq \lim _{N \rightarrow \infty} \widehat{\theta}\left(\left(A^{*}\right)^{-N} \mathbf{t}\right) \leq 1
$$

(iii) $\lim _{N \rightarrow \infty} \widehat{\theta}\left(\left(A^{*}\right)^{-N} \mathbf{t}\right)$ is either 0 or 1 a.e. on $\mathbb{R}^{n}$. Moreover, the first case occurs if and only if $\widehat{\theta}\left(\left(A^{*}\right)^{-N} \mathbf{t}\right)=0$ for each $N \in \mathbb{Z}$.

Proof. (i) is an immediate consequence of the definition of $\widehat{\theta}$ and the fact that $|H(\mathbf{t})| \leq 1$ a.e. on $\mathbb{R}^{n}$.

(ii) follows from the fact that $0 \leq \widehat{\theta}(\mathbf{t}) \leq 1$ a.e. on $\mathbb{R}^{n}$ and from the monotonicity expressed in (i).

To show (iii) observe that by (ii), $\lim _{N \rightarrow \infty} \widehat{\theta}\left(\left(A^{*}\right)^{-N} \mathbf{t}\right)$ exists for all $\mathbf{t} \in$ $\mathbb{R}^{n} \backslash G$ where $G \subset \mathbb{R}^{n}$ is a measurable set such that $|G|_{n}=0$. Moreover, if we set $F=\left\{\mathbf{t} \in \mathbb{R}^{n}:\left|H\left(\left(A^{*}\right)^{N} \mathbf{t}\right)\right|>1\right.$ for some $\left.N \in \mathbb{Z}\right\}$, then from hypothesis, $|F|_{n}=0$.

Given $\mathbf{t} \in \mathbb{R}^{n} \backslash G$, it is obvious that if $\widehat{\theta}\left(A^{* N} \mathbf{t}\right)=0$ for all $N \in \mathbb{Z}$, then $\lim _{N \rightarrow \infty} \widehat{\theta}\left(\left(A^{*}\right)^{-N} \mathbf{t}\right)=0$. On the other hand, given $\mathbf{t} \in \mathbb{R}^{n} \backslash(G \cup F)$, if there exists an $N_{0} \in \mathbb{Z}$ such that $\widehat{\theta}\left(A^{*-N_{0}} \mathbf{t}\right) \neq 0$ we have

$$
0<\widehat{\theta}\left(\left(A^{*}\right)^{-N_{0}} \mathbf{t}\right)=\prod_{j=1}^{\infty}\left|H\left(\left(A^{*}\right)^{-j-N_{0}} \mathbf{t}\right)\right|=\prod_{j=N_{0}+1}^{\infty}\left|H\left(\left(A^{*}\right)^{-j} \mathbf{t}\right)\right| .
$$

Thus

$$
\prod_{j=N_{0}+1}^{N}\left|H\left(\left(A^{*}\right)^{-j} \mathbf{t}\right)\right|>0 \quad \forall N \geq N_{0}+1 .
$$

Hence, when $N \geq N_{0}+1$ we have

$$
\widehat{\theta}\left(\left(A^{*}\right)^{-N_{0}} \mathbf{t}\right)=\prod_{j=N_{0}+1}^{N}\left|H\left(\left(A^{*}\right)^{-j} \mathbf{t}\right)\right| \widehat{\theta}\left(\left(A^{*}\right)^{-N} \mathbf{t}\right)>0,
$$

and consequently, as $\left\{\prod_{j=N_{0}+1}^{N}\left|H\left(\left(A^{*}\right)^{-j} \mathbf{t}\right)\right|\right\}_{N=N_{0}+1}^{\infty}$ is a nonincreasing sequence such that

$$
\lim _{N \rightarrow \infty} \prod_{j=N_{0}+1}^{N}\left|H\left(\left(A^{*}\right)^{-j} \mathbf{t}\right)\right|=\widehat{\theta}\left(\left(A^{*}\right)^{-N_{0}} \mathbf{t}\right),
$$

we obtain

$$
\lim _{N \rightarrow \infty} \widehat{\theta}\left(\left(A^{*}\right)^{-N} \mathbf{t}\right)=\lim _{N \rightarrow \infty} \frac{\widehat{\theta}\left(\left(A^{*}\right)^{-N_{0}} \mathbf{t}\right)}{\prod_{j=N_{0}+1}^{N}\left|H\left(\left(A^{*}\right)^{-j} \mathbf{t}\right)\right|}=1 .
$$


The following corollary is a consequence of Proposition 7 .

Corollary 1. Let $H \in L^{\infty}\left(\mathbb{T}^{n}\right)$ be such that (3) holds and let $\widehat{\theta}(\mathbf{t})=$ $\prod_{j=1}^{\infty}\left|H\left(\left(A^{*}\right)^{-j} \mathbf{t}\right)\right|$ a.e. on $\mathbb{R}^{n}$. Then either $\widehat{\theta}$ is not $A^{*}$-locally nonzero at the origin or the origin is a point of $A^{*}$-approximate continuity of $\widehat{\theta}$ if we set $\widehat{\theta}(\mathbf{0})=1$.

Proof. It is enough to prove that if $\widehat{\theta}$ is $A^{*}$-locally nonzero at the origin then the origin is a point of $A^{*}$-approximate continuity of $\widehat{\theta}$ if we set $\widehat{\theta}(\mathbf{0})=1$. According to our hypothesis, by Proposition 4 there exists a measurable set $G \subset \mathbb{R}^{n}$ with $|G|_{n}=0$ and an increasing sequence $\left\{N_{k}\right\}_{k=1}^{\infty} \subset \mathbb{N}$ such that for every $\mathbf{t} \in \mathbb{R}^{n} \backslash G$ there exists $k_{0} \in \mathbb{N}$ such that if $k \geq k_{0}$, then $\widehat{\theta}\left(\left(A^{*}\right)^{-N_{k}} \mathbf{t}\right)$ $\neq 0$. Thus from condition (iii) of Proposition $7, \lim _{N \rightarrow \infty}\left|\widehat{\theta}\left(A^{-N} \mathbf{t}\right)\right|=1$ for all $\mathbf{t} \in \mathbb{R}^{n} \backslash G$. Hence, an application of Proposition 1 finishes the proof.

5. Proof of Theorem 1. Let us begin with the proof of the implication $(\mathrm{A}) \Rightarrow(\mathrm{B})$. That $f \equiv 1$ is invariant under $P$ is an immediate consequence of Proposition B.

Suppose that $f \in L^{1}\left(\mathbb{T}^{n}\right) \cap \Pi_{A}$ is a fixed point of the operator $P$. We will show that

$$
\int_{[0,1]^{n}} f(\mathbf{t}) d \mathbf{t} \geq 1 .
$$

This condition together with $f \in \Pi_{A}$ will show that $f \equiv 1$.

Using the equality $P f=f$, we obtain

$$
\begin{aligned}
\int_{[0,1]^{n}} f(\mathbf{t}) d \mathbf{t} & =\int_{[0,1]^{n}} P(f)(\mathbf{t}) d \mathbf{t} \\
& =\int_{[0,1]^{n}} \sum_{i=0}^{d_{A}-1}\left|H\left(\left(A^{*}\right)^{-1}\left(\mathbf{t}+\mathbf{p}_{i}\right)\right)\right|^{2} f\left(\left(A^{*}\right)^{-1}\left(\mathbf{t}+\mathbf{p}_{i}\right)\right) d \mathbf{t} \\
& =d_{A} \int_{[0,1]^{n}}|H(\mathbf{t})|^{2} f(\mathbf{t}) d \mathbf{t}=d_{A} \int_{[-1 / 2,1 / 2]^{n}}|H(\mathbf{t})|^{2} f(\mathbf{t}) d \mathbf{t},
\end{aligned}
$$

where the third equality follows from Lemma $\mathrm{D}(\mathrm{ii})$, and the last equality is true because $H$ and $f$ are $\mathbb{Z}^{n}$-periodic functions.

Putting $A^{*} \mathbf{t}=\mathbf{v}$, we obtain

$$
\begin{aligned}
\int_{[0,1]^{n}} f(\mathbf{t}) d \mathbf{t} & =\int_{\mathbb{R}^{n}}\left|H\left(\left(A^{*}\right)^{-1} \mathbf{v}\right)\right|^{2} f\left(\left(A^{*}\right)^{-1} \mathbf{v}\right) \chi_{[-1 / 2,1 / 2]^{n}}\left(\left(A^{*}\right)^{-1} \mathbf{v}\right) d \mathbf{v} \\
& =\int_{\mathbb{R}^{n}}\left|H\left(\left(A^{*}\right)^{-1} \mathbf{t}\right)\right|^{2} \operatorname{Pf}\left(\left(A^{*}\right)^{-1} \mathbf{t}\right) \chi_{[-1 / 2,1 / 2]^{n}}\left(\left(A^{*}\right)^{-1} \mathbf{t}\right) d \mathbf{t},
\end{aligned}
$$

since $P f=f$. 
Repeating the above calculations and using the condition $A^{*}\left(\mathbb{Z}^{n}\right) \subset \mathbb{Z}^{n}$, we obtain

$$
\int_{[0,1]^{n}} f(\mathbf{t}) d \mathbf{t}=\int_{\mathbb{R}^{n}} \prod_{j=1}^{N}\left|H\left(\left(A^{*}\right)^{-j} \mathbf{t}\right)\right|^{2} f\left(\left(A^{*}\right)^{-N} \mathbf{t}\right) \chi_{[-1 / 2,1 / 2]^{n}}\left(\left(A^{*}\right)^{-N} \mathbf{t}\right) d \mathbf{t} .
$$

Let

$$
\Gamma_{N} f(\mathbf{t})=\prod_{j=1}^{N}\left|H\left(\left(A^{*}\right)^{-j} \mathbf{t}\right)\right|^{2} f\left(\left(A^{*}\right)^{-N} \mathbf{t}\right) \chi_{[-1 / 2,1 / 2]^{n}}\left(\left(A^{*}\right)^{-N} \mathbf{t}\right) \quad \text { for } N \in \mathbb{N} .
$$

Since the origin is a point of $A^{*}$-approximate continuity of $f$, it is a point of $A^{*}$-approximate continuity of $\chi_{[-1 / 2,1 / 2]^{n}} f$. Hence, according to Proposition 3 , there exists an increasing sequence $\left\{l_{N}\right\}_{N=1}^{\infty} \subset \mathbb{N}$ such that

$$
\lim _{N \rightarrow \infty} \Gamma_{l_{N}} f(\mathbf{t})=\prod_{j=1}^{\infty}\left|H\left(\left(A^{*}\right)^{-j} \mathbf{t}\right)\right|^{2} \quad \text { a.e. on } \mathbb{R}^{n} .
$$

By Fatou's lemma and (8),

$$
\begin{aligned}
\int_{[0,1]^{n}} f(\mathbf{t}) d \mathbf{t} & =\lim _{N \rightarrow \infty} \int_{\mathbb{R}^{n}} \Gamma_{l_{N}} f(\mathbf{t}) d \mathbf{t} \geq \int_{\mathbb{R}^{n}} \lim _{N \rightarrow \infty} \Gamma_{l_{N}} f(\mathbf{t}) d \mathbf{t} \\
& =\int_{\mathbb{R}^{n}} \prod_{j=1}^{\infty}\left|H\left(\left(A^{*}\right)^{-j} \mathbf{t}\right)\right|^{2} d \mathbf{t}=\int_{\mathbb{R}^{n}}|\widehat{\theta}(\mathbf{t})|^{2} d \mathbf{t}=1 .
\end{aligned}
$$

To prove $(\mathrm{B}) \Rightarrow(\mathrm{A})$, first observe that we can redefine $H$ in a set of null measure so that (3) holds for all $\mathbf{t} \in \mathbb{R}^{n}$. Indeed, if $G \subset \mathbb{T}^{n}$ with $|G|_{n}=0$ is the exceptional set where (3) does not hold, then $G=\bigcup_{i=0}^{d_{A}-1}\left(G+\left(A^{*}\right)^{-1} \mathbf{p}_{i}\right)$. We set $|H(\mathbf{t})|=1 / \sqrt{d_{A}}$ for $\mathbf{t} \in G$. By Proposition C, we have $\widehat{\theta} \in L^{2}\left(\mathbb{R}^{n}\right)$.

We now show that the function $\Phi_{\theta}$ defined by (1) is a fixed point for $P$. We have

$$
\begin{aligned}
\Phi_{\theta}(\mathbf{t}) & =\sum_{\mathbf{k} \in \mathbb{Z}^{n}}|\widehat{\theta}(\mathbf{t}+\mathbf{k})|^{2}=\sum_{i=0}^{d_{A}-1} \sum_{\mathbf{k} \in \mathbf{p}_{i}+A^{*} \mathbb{Z}^{n}}|\widehat{\theta}(\mathbf{t}+\mathbf{k})|^{2} \\
& =\sum_{i=0}^{d_{A}-1} \sum_{\mathbf{q} \in \mathbb{Z}^{n}}\left|\widehat{\theta}\left(\mathbf{t}+\mathbf{p}_{i}+A^{*} \mathbf{q}\right)\right|^{2} .
\end{aligned}
$$

Hence, from the definition of $\widehat{\theta}$, we obtain

$$
\begin{aligned}
\Phi_{\theta}(\mathbf{t}) & =\sum_{i=0}^{d_{A}-1} \sum_{\mathbf{q} \in \mathbb{Z}^{n}}\left|H\left(\left(A^{*}\right)^{-1} \mathbf{t}+\left(A^{*}\right)^{-1} \mathbf{p}_{i}+\mathbf{q}\right)\right|^{2}\left|\widehat{\theta}\left(\left(A^{*}\right)^{-1} \mathbf{t}+\left(A^{*}\right)^{-1} \mathbf{p}_{i}+\mathbf{q}\right)\right|^{2} \\
& =\sum_{i=0}^{d_{A}-1}\left|H\left(\left(A^{*}\right)^{-1} \mathbf{t}+\left(A^{*}\right)^{-1} \mathbf{p}_{i}\right)\right|^{2} \Phi_{\theta}\left(\left(A^{*}\right)^{-1} \mathbf{t}+\left(A^{*}\right)^{-1} \mathbf{p}_{i}\right)=P\left(\Phi_{\theta}\right)(\mathbf{t})
\end{aligned}
$$

a.e. on $\mathbb{R}^{n}$, because $H$ is $\mathbb{Z}^{n}$-periodic. 
If we prove that $\Phi_{\theta} \in L^{1}\left(\mathbb{T}^{n}\right) \cap \Pi_{A}$, then $\Phi_{\theta}(\mathbf{t})=1$ a.e. on $\mathbb{T}^{n}$ by condition (B) of Theorem 1. Hence by Theorem A, $\theta$ is a scaling function of an $A$-MRA with associated low pass filter $H$, and the proof of Theorem 1 will be finished.

Obviously, $0 \leq \Phi_{\theta}(\mathbf{t})$ a.e. on $\mathbb{R}^{n}$ and $\Phi_{\theta}$ is a $\mathbb{Z}^{n}$-periodic function.

We define, for every $N \in \mathbb{N}$, a function $\Gamma_{N}: \mathbb{R}^{n} \rightarrow[0,1]$ by

$$
\Gamma_{N}(\mathbf{t})=\prod_{j=1}^{N}\left|H\left(\left(A^{*}\right)^{-j} \mathbf{t}\right)\right| \chi_{[-1 / 2,1 / 2]^{n}}\left(\left(A^{*}\right)^{-N} \mathbf{t}\right), \quad \mathbf{t} \in \mathbb{R}^{n} .
$$

For any $\mathbf{t} \in \mathbb{R}^{n}$, there exists an $N_{0} \in \mathbb{N}$ such that $\mathbf{t} \in A^{* N}[-1 / 2,1 / 2]^{n}$ for all $N \geq N_{0}$. The sequence of numbers $\left\{\Gamma_{N}(\mathbf{t})\right\}_{N=N_{0}}^{\infty}$ is nonincreasing, and also the sequence of functions $\left\{\Gamma_{N}(\mathbf{t})\right\}_{N=1}^{\infty}$ converges everywhere and the limit coincides with the function $\widehat{\theta}(\mathbf{t})$ a.e. on $\mathbb{R}^{n}$.

Hence

$$
\begin{aligned}
\operatorname{ess~sup}_{\mathbf{t} \in[-1 / 2,1 / 2]^{n}} \Phi_{\theta}(\mathbf{t}) & =\lim _{N \rightarrow \infty} \operatorname{esssup}_{\mathbf{t} \in[-1 / 2,1 / 2]^{n}} \sum_{\substack{\mathbf{k} \in \mathbb{Z}^{n} \\
\mathbf{k} \in[-N, N]^{n}}}|\widehat{\theta}(\mathbf{t}+\mathbf{k})|^{2} \\
& \leq \lim _{N \rightarrow \infty} \operatorname{ess~sup}_{\mathbf{t} \in[-1 / 2,1 / 2]^{n}} \sum_{\substack{\mathbf{k} \in \mathbb{Z}^{n} \\
\mathbf{k} \in[-N, N]^{n}}}\left|\Gamma_{L_{N}}(\mathbf{t}+\mathbf{k})\right|^{2} \\
& \leq \lim _{N \rightarrow \infty} \operatorname{ess~sup}_{\mathbf{t} \in[-1 / 2,1 / 2]^{n}} \sum_{\mathbf{k} \in \mathbb{Z}^{n}}\left|\Gamma_{L_{N}}(\mathbf{t}+\mathbf{k})\right|^{2}=1
\end{aligned}
$$

by Lemma $\mathrm{E}$, where $L_{N} \in \mathbb{N}$ is such that $\mathbf{t}+\mathbf{k} \in A^{* L_{N}}[-1 / 2,1 / 2]^{n}$ for all $\mathbf{t} \in[-1 / 2,1 / 2]^{n}$ and all $\mathbf{k} \in[-N, N]^{n}$.

It remains to prove that the origin is a point of $A^{*}$-approximate continuity of $\Phi_{\theta}$ if we set $\Phi_{\theta}(\mathbf{0})=1$. By hypothesis, $\widehat{\theta}$ is $A^{*}$-locally nonzero at the origin, thus according to Corollary 1 , the origin is a point of $A^{*}$-approximate continuity of $\widehat{\theta}$ if we set $\widehat{\theta}(\mathbf{0})=1$. Hence, the inequalities $\widehat{\theta}(\mathbf{t}) \leq \Phi_{\theta}(\mathbf{t}) \leq 1$ yield the required assertion.

6. Proof of Theorem 2. Let $A: \mathbb{R}^{n} \rightarrow \mathbb{R}^{n}$ be a linear invertible map such that $A\left(\mathbb{Z}^{n}\right) \subset \mathbb{Z}^{n}$ and all (complex) eigenvalues of $A$ have modulus greater than 1. Suppose that $m$ is a $\mathbb{Z}^{n}$-periodic function and $|m(\mathbf{t})|=1$ a.e. on $\mathbb{R}^{n}$. We claim that there exists a measurable function $\mu: \mathbb{R}^{n} \rightarrow \mathbb{C}$ such that $|\mu(\mathbf{t})|=1$ a.e. on $\mathbb{R}^{n}$ and

$$
m(\mathbf{t})=\mu\left(A^{*} \mathbf{t}\right) \overline{\mu(\mathbf{t})} .
$$

We set $F=B_{1} \backslash \bigcup_{j=1}^{\infty}\left(A^{*}\right)^{-j} B_{1}$, and observe that $|F|_{n}>0$. We know that $d_{A}$ is a natural number, hence $d_{A} \geq 2$ and 


$$
\left|\bigcup_{j=1}^{\infty}\left(A^{*}\right)^{-j} B_{1}\right|_{n}<\sum_{j=1}^{\infty} d_{A}^{-j}\left|B_{1}\right|_{n}=\frac{1}{d_{A}-1}\left|B_{1}\right|_{n} \leq\left|B_{1}\right|_{n}
$$

because any set $\left(A^{*}\right)^{-j} B_{1}$ contains a neighbourhood of the origin.

Next, observe that

$$
A^{* j} F \cap A^{* i} F=\emptyset \quad \text { if } j, i \in \mathbb{Z} \text { and } i \neq j .
$$

If $\mathbf{x} \in \mathbb{R}^{n} \backslash\{\mathbf{0}\}$, as all (complex) eigenvalues of $A$ have modulus greater than 1 , there exists $N \in \mathbb{Z}$ such that $\mathbf{x} \in\left(A^{*}\right)^{-N} B_{1}$ and $\mathbf{x} \notin \bigcup_{j=N+1}^{\infty}\left(A^{*}\right)^{-j} B_{1}$. Thus $\mathbf{x} \in\left(A^{*}\right)^{-N} B_{1} \backslash \bigcup_{j=N+1}^{\infty}\left(A^{*}\right)^{-j} B_{1}=\left(A^{*}\right)^{-N} F$, so $\bigcup_{j=-\infty}^{\infty} A^{* j} F=$ $\mathbb{R}^{n} \backslash\{\mathbf{0}\}$.

Now we are prepared to construct a measurable function $\mu$ such that $|\mu(\mathbf{t})|=1$ a.e. on $\mathbb{R}^{n}$ and (9) holds. First, we define a measurable function $\mu$ on $F$ such that $|\mu(\mathbf{t})|=1$ if $\mathbf{t} \in F$. From (10), if $\mathbf{x} \in A^{*} F$, we put

$$
\mu(\mathbf{x})=m\left(\left(A^{*}\right)^{-1} \mathbf{x}\right) \mu\left(\left(A^{*}\right)^{-1} \mathbf{x}\right),
$$

and thus (9) is satisfied for $\mathbf{t} \in F$. Afterwards, step by step we can define $\mu$ on the sets $F_{N}=\bigcup_{j=0}^{N} A^{* j} F$, so that (9) is valid on $F_{N}$.

In an analogous way, if $\mathbf{t} \in\left(A^{*}\right)^{-1} F$ we can define

$$
\mu(\mathbf{t})=\mu\left(A^{*} \mathbf{t}\right) \overline{m(\mathbf{t})},
$$

and then (9) will be true for $\mathbf{t} \in\left(A^{*}\right)^{-1} F$. Then again step by step we can define $\mu$ on the sets $E_{N}=\bigcup_{j=1}^{N}\left(A^{*}\right)^{-j} F$, so that (9) holds on $E_{N}$, and thus finish the construction.

Let $H$ be a low pass filter associated with the scaling function $\phi$ of an $A$-MRA. We claim that $\widetilde{H}=m H$ is the low pass filter associated with the scaling function $\widetilde{\phi}$ where $\widehat{\widetilde{\phi}}=\mu \widehat{\phi}$. Let us check the conditions of Theorem A for $\widetilde{\phi}$. It is clear that $(\alpha)$ and $(\beta)$ are true. Moreover,

$$
\begin{aligned}
\widehat{\widetilde{\phi}}\left(A^{*} \mathbf{t}\right) & =\mu\left(A^{*} \mathbf{t}\right) \widehat{\phi}\left(A^{*} \mathbf{t}\right)=\mu\left(A^{*} \mathbf{t}\right) H(\mathbf{t}) \widehat{\phi}(\mathbf{t}) \\
& =\mu\left(A^{*} \mathbf{t}\right) \overline{\mu(\mathbf{t})} H(\mathbf{t}) \mu(\mathbf{t}) \widehat{\phi}(\mathbf{t})=m(\mathbf{t}) H(\mathbf{t}) \widehat{\widetilde{\phi}}(\mathbf{t})=\widetilde{H}(\mathbf{t}) \widehat{\widetilde{\phi}}(\mathbf{t}),
\end{aligned}
$$

and thus $(\gamma)$ holds for $\tilde{\phi}$.

To prove the necessity, we suppose that $m$ is a filter multiplier. Take a low pass filter $H_{h}$ which is almost everywhere nonzero; it exists by Claim 1. Since $m^{k} H_{h}$ is also a low pass filter for any $k \in \mathbb{N}$, it must satisfy condition (3). Consequently, by letting $k \rightarrow \infty$, we see that $|m(\mathbf{t})| \leq 1$ a.e. on $\mathbb{R}^{n}$. Otherwise, $\left|m^{k} H_{h}\right|$ would be larger than 1 for some big $k$ on a set of positive measure, which is impossible. Likewise, $|m|$ cannot be smaller than 1 on a set of positive measure, since this would contradict (3) for $m H_{h}$. 
Since $H_{h}(\mathbf{t}) \neq 0$ a.e. on $\mathbb{R}^{n}$, and $H:=m H_{h}$ is a low pass filter of an $A$-MRA, the function $m(\mathbf{t})=H(\mathbf{t}) / H_{h}(\mathbf{t})$ is well defined a.e. on $\mathbb{R}^{n}$ as a $\mathbb{Z}^{n}$-periodic function.

Acknowledgements. The author is grateful to Professors Kazaros Kazarian and Patrico Cifuentes for useful discussions and help in preparing the final version of this paper. Moreover, the author is thankful to anonymous referees whose suggestions improved the presentation of this paper.

\section{References}

[1] C. Boor, R. DeVore and A. Ron, On the construction of multivariate (pre)wavelets, Constr. Approx. 9 (1993), 123-166.

[2] M. Bownik, Tight frames of multidimensional wavelets, J. Fourier Anal. Appl. 3 (1997), 525-542.

[3] A. Bruckner, Differentiation of Real Functions, Lecture Notes in Math. 659, Springer, Berlin, 1978.

[4] P. Cifuentes, K. S. Kazarian and A. San Antolín, Characterization of scaling functions in a multiresolution analysis, Proc. Amer. Math. Soc. 133 (2005), 1013-1023.

[5] A. Cohen, Ondelettes, analyses multirésolutions et filtres miroirs en quadrature, Ann. Inst. H. Poincaré Anal. Non Linéaire 7 (1990), 439-459.

[6] A. Cohen, I. Daubechies and J. C. Feauveau, Biorthogonal bases of compactly supported wavelets, Comm. Pure Appl. Math. 45 (1992), 485-560.

[7] S. Dahlke, W. Dahmen and V. Latour, Smooth refinable functions and wavelets obtained by convolution products. Appl. Comput. Harmonic Anal. 2 (1995), 68-84.

[8] I. Daubechies, Ten Lectures on Wavelets, SIAM, Philadelphia, 1992.

[9] V. Dobrić, R. F. Gundy and P. Hitczenko, Characterizations of orthonormal scale functions: a probabilistic approach, J. Geom. Anal. 10 (2000), 417-434.

[10] K. Gröchenig and W. R. Madych, Multiresolution analysis, Haar bases and selfsimilar tillings of $R^{n}$, IEEE Trans. Inform. Theory 38 (1992), 556-568.

[11] R. F. Gundy, Two remarks concerning wavelets: Cohen's criterion for low-pass filters and Meyer's theorem on linear independence, in: The Functional and Harmonic Analysis of Wavelets and Frames (San Antonio, TX, 1999), Contemp. Math. 247, Amer. Math. Soc., Providence, RI, 1999, 249-258.

[12] —, Low-pass filters, martingales, and multiresolution analyses, Appl. Comput. Harmon. Anal. 9 (2000), 204-219.

[13] E. Hernández and G. Weiss, A First Course on Wavelets, CRC Press, 1996.

[14] L. Hörmander, The Analysis of Linear Partial Differential Operators. I. Distribution Theory and Fourier Analysis, Grundlehren Math. Wiss. 256, Springer, Berlin, 1983.

[15] R. Q. Jia and C. A. Micchelli, Using the refinement equations for the construction of pre-wavelets. II. Powers of two, in: Curves and Surfaces (Chamonix-Mont-Blanc, 1990), Academic Press, Boston, MA, 1991, 209-246.

[16] W. M. Lawton, Tight frames of compactly supported affine wavelets, J. Math. Phys. 31 (1990), 1898-1901.

[17] - Necessary and sufficient conditions for constructing orthonormal wavelet bases, J. Math. Phys. 32 (1991), 57-61. 
[18] R. A. Lorentz, W. R. Madych and A. Sahakian, Translation and dilation invariant subspaces of $L^{2}(\mathbf{R})$ and multiresolution analyses, Appl. Comput. Harmonic Anal. 5 (1998), 375-388.

[19] W. R. Madych, Some elementary properties of multiresolution analyses of $L^{2}\left(R^{d}\right)$, in: Wavelets - a Tutorial in Theory and Applications, Ch. Chui (ed.), Academic Press, 1992, 259-294.

[20] S. Mallat, Multiresolution approximations and wavelet orthonormal bases for $L^{2}(R)$, Trans. Amer. Math. Soc. 315 (1989), 69-87.

[21] Y. Meyer, Ondelettes et opérateurs. I, Hermann, Paris, 1990; English transl.: Wavelets and Operators, Cambridge Univ. Press, 1992.

[22] I. P. Natanson, Theory of Functions of a Real Variable, Vol. II, London, 1960.

[23] M. Papadakis, H. Sikić and G. Weiss, The characterization of low pass filters and some basic properties of wavelets, scaling functions and related concepts, J. Fourier Anal. Appl. 5 (1999), 495-521.

[24] R. Strichartz, Construction of orthonormal wavelets, in: Wavelets: Mathematics and Applications, Stud. Adv. Math., CRC Press, Boca Raton, FL, 1994, 23-50.

[25] P. Wojtaszczyk, A Mathematical Introduction to Wavelets, London Math. Soc. Student Texts 37, Cambridge Univ. Press, 1997.

[26] The Wutam Consortium, Basic properties of wavelets, J. Fourier Anal. Appl. 4 (1998), 575-594.

Departamento de Matemáticas

Universidad Autónoma de Madrid

28049 Madrid, Spain

E-mail: angel.sanantolin@uam.es

Received May 16, 2007

Revised version June 16, 2008 